\title{
Case Report \\ Pediatric Cryptococcal Lymphadenitis in the Absence of AIDS: Case Report and Literature Review
}

\author{
Fengchang Bao, ${ }^{1}$ Hongna Tan, ${ }^{2}$ Wei Liu, ${ }^{1}$ Yange $\mathrm{Li}^{1},{ }^{1}$ and Huixia $\mathrm{Li}^{1}$ \\ ${ }^{1}$ Department of Hematology, Children's Hospital, Zhengzhou, 255 Gangdu Road, Henan 450052, China \\ ${ }^{2}$ Department of Radiology, The First Affilicated Hospital of Zhengzhou University, Zhengzhou, 1 Jianshe East Road, \\ Henan 450000, China \\ Correspondence should be addressed to Wei Liu; liuweixinxiang123@163.com
}

Received 20 March 2013; Accepted 29 April 2013

Academic Editors: C. F. Classen, J. Kobr, C. Modesto, I. Riaño Galán, and A. T. Soliman

Copyright (C) 2013 Fengchang Bao et al. This is an open access article distributed under the Creative Commons Attribution License, which permits unrestricted use, distribution, and reproduction in any medium, provided the original work is properly cited.

\begin{abstract}
We present a rare case of cryptococcal lymphadenitis without immunocompromization in a two-and-a-half-year-old child. He was referred to our center with a fifteen-day history of continued fever. Ultrasound and computed tomography (CT) revealed the enlargement of multiple lymph nodes and lung reticulonodular shadows. Hematological, immunological, and microbiological tests for hepatitis, lymphoma, AIDS, and immunoglobulin deficiencies were negative. Laboratory tests demonstrated elevated erythrocyte sedimentation rate, elevated plasma and urinary $ß 2$-microglobulin (ß2-MG) levels, and elevated C-reactive protein and fibrinogen. Both blood routine and bone marrow aspiration showed elevated eosinophil granulocytes. The diagnosis of cryptococcal lymphadenitis was obtained by excisional biopsy of the cervical lymph nodes. The patient was treated with intravenous amphotericin $\mathrm{B}$ and oral flucytosine for five weeks, then with subsequent oral fluconazole for three months. The patient is now doing well. Our case suggests that the diagnosis of cryptococcal lymphadenitis is very difficult without etiology and pathology, especially for a patient with a normal immune system; lymph node biopsy is necessary to diagnose it, and immediate antifungal treatment is necessary to treat it.
\end{abstract}

\section{Introduction}

Cryptococcal lymphadenitis occurs commonly as an opportunistic infection in AIDS patients and may be life threatening $[1-8]$. Kim et al. reported one case of this infection in a patient with systemic lupus erythematosus [9]. This infection has several clinical but nonspecific manifestations, including pneumonia, meningitis, peritonitis, and disseminated infection. Cryptococcal lymphadenitis in the absence of AIDS is extremely rare, and it is difficult to distinguish from other lymphadenopathies such as lymphoma and tuberculosis. In the present case, we describe a pediatric case of cryptococcal lymphadenitis in a patient who did not have AIDS and who presented with multiple cervical and retroperitoneal lymph node enlargements and pulmonary inflammatory lesions. This case was misdiagnosed as lymphoma, and the final diagnosis was confirmed by cervical lymph node excisional biopsy.

\section{Case Presentation}

A two-and-a-half-year-old child was referred to our center with a fifteen-day history of fever without an obvious remote cause. The child presented with a continued fever, with a maximum temperature of 40 degrees and without shivering, nausea, emesia, and hyperspasmia. The child was treated with two weeks of standard antibiotic treatment for pneumonia (indicated by a chest X-ray) in the local hospital before he came to our center; however, the fever was not controlled. At the time of his presentation, the child was fully conscious, and a history of contact with HIV was denied by his parents. Physical examination showed bilateral multiple cervical lymph node enlargements, as well as mild hepatosplenomegaly. A diagnosis of lymphoma was suspected by the clinician.

Detailed examinations were performed after he came to our center. Ultrasonography revealed multiple retroperitoneal lymph node enlargements (Figure 1). An enhanced 


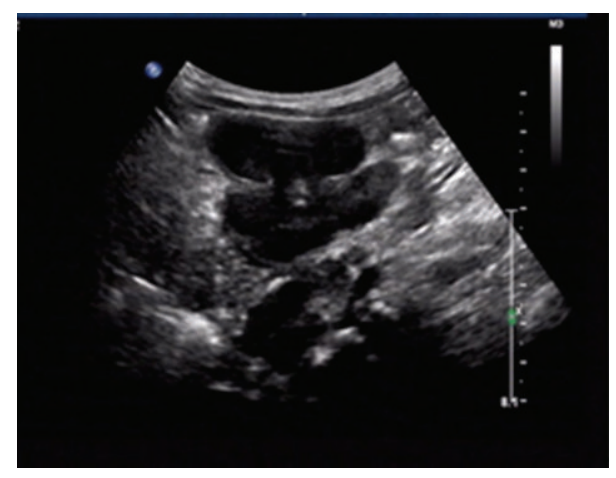

FIGURE 1: Ultrasound demonstrates multiple retroperitoneal lymph node enlargements.

64-slice thoracic and abdominal computerized tomography (CT) scan was obtained, which revealed multiple lymph node enlargements in the bilateral neck, lung hilum, mediastinum, and retroperitoneal area (Figure 2), and some of the lymph nodes had fused into a mass and appeared as having a moderately homogeneous enhancement. At the same time, the thoracic CT scan also revealed reticulonodular shadows in the lung (Figure 3(a)). The ultrasound and CT findings could not exclude a diagnosis of lymphoma. Cryptococcus neoformans was not found by India ink stain of the cerebrospinal fluid. Hematological, immunological, and microbiological tests for hepatitis, lymphoma, AIDS, and immunoglobulin deficiencies were negative. Laboratory tests demonstrated an elevated erythrocyte sedimentation rate $(20 \mathrm{~mm} / \mathrm{h})$, elevated plasma and urinary $\beta 2-\mathrm{MG}$ levels $(4.2 \mu \mathrm{g} / \mathrm{mL}$ and $0.44 \mu \mathrm{g} / \mathrm{mL}$, resp.), elevated C-reactive protein $(153.92 \mathrm{mg} / \mathrm{L})$, and elevated fibrinogen $(6.73 \mathrm{~g} / \mathrm{L})$. Blood routine showed elevated WBC $\left(44.22 \times 10^{9}\right)$, neutrophil granulocytes (74.24\%), eosinophil granulocytes (7.14\%), and absolute counting of eosinophil granulocytes $\left(3.13 \times 10^{9}\right)$. Bone marrow aspiration showed elevated eosinophil granulocytes (10.5\%), and peripheral blood lymphocyte subset detection by flow cytometry showed that the percentages of $\mathrm{CD} 3, \mathrm{CD} 3 / \mathrm{CD}^{+}, \mathrm{CD} 3 / \mathrm{CD} 4^{+}, \mathrm{CD} 9^{+}$, and NK were $54 \%, 9 \%$, $42 \%, 42 \%$, and $2 \%$, respectively (Figure 4 ). Finally, a cervical lymph node excisional biopsy was performed, revealing a lymphohistiocytic background with several blue, spherical structures surrounded by clear halos, suggestive of Cryptococcus (Figure 5). The yeast-like structures were identified by periodic acid-Schiff (PAS) stain. After this patient was treated with intravenous amphotericin B $(0.7 \mathrm{mg} / \mathrm{kg} /$ day $)$ and oral flucytosine $(100 \mathrm{mg} / \mathrm{kg} /$ day $)$ for five weeks, the lung lesions and lymph nodes were markedly diminished in size (Figures 3 (b) and 6). Subsequent oral fluconazole (12 mg/kg/day) was administered for three months, and the patient is now doing well.

\section{Discussion}

Cryptococcosis is a subacute to chronic infection caused by the encapsulated yeast Cryptococcus neoformans, which can involve the respiratory system, central nervous system,

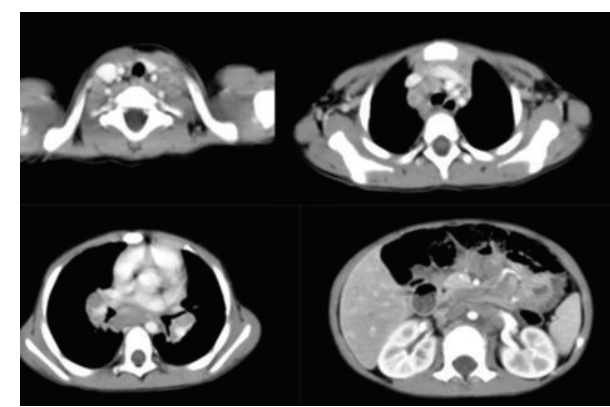

FIgURE 2: Thoracic and abdominal CT shows multiple lymph node enlargements in the regions of the bilateral neck and hilum, as well as the mediastinum and retroperitoneal areas.

skin, lymph nodes, and other organs. Approximately 85\% of patients with cryptococcosis have impaired cell-mediated immunity. AIDS-associated cryptococcal infections now account for $80-90 \%$ of all patients with cryptococcosis [10]. Cryptococcus neoformans is distributed worldwide; it can be inhaled into the respiratory tract, and depending on the immune response, the host may be asymptomatic or the disease may present as pneumonitis, pulmonary nodules, meningitis (if it disseminates to the CNS), or, less commonly, lymph node enlargement. Therefore, the respiratory tract and CNS are the primary sites of infection, with occasional involvement of the lymph nodes [11]. Cryptococcal lymphadenitis occurs more commonly in immunocompromised patients $[5,6,8,9]$. Mitha et al. reported that HIVnegative patients generally present with pulmonary or CNS mass lesions, whereas meningoencephalitis is predominant in HIV-positive patients [11]. In our case, a pediatric HIVnegative patient with cryptococcal lymphadenitis presented with persistent high fever without obvious CNS symptoms, which is in agreement with previous studies. Although multiple lymph node enlargements are unusual in HIVnegative cryptococcosis, this phenomenon may be related to the patient's age. Our patient was two-and-a-half-year old, so it is possible that his immune system was not fully developed. To the best of our knowledge, this finding has not been previously reported.

The clinical appearances and laboratory tests for cryptococcal lymphadenitis are nonspecific. Indirect evidence of infection by detection of cryptococcal antigens is particularly helpful because the antigens have high sensitivity and specificity, but negative results do not absolutely rule out cryptococcosis because there may be only a small number of organisms present. In our patient, ultrasound and CT findings clearly demonstrated swollen lymph nodes regardless of neck or retroperitoneal, and thoracic CT also revealed the lung diseases. However, it was very difficult to distinguish cryptococcal lymphadenitis from lymphoma and tuberculosis in our case, despite the laboratory test results (elevated erythrocyte sedimentation rate, plasma and urinary $\beta 2-\mathrm{MG}$ levels, and C-reactive protein and fibrinogen) [1215]. Therefore, the diagnosis of cryptococcal lymphadenitis is generally based on lymph node biopsy $[16,17]$. Histopathology will show cryptococcus as thin-walled, dark-staining cells 


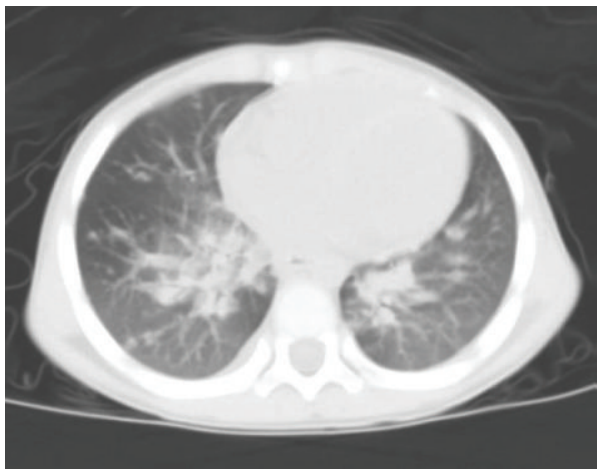

(a)

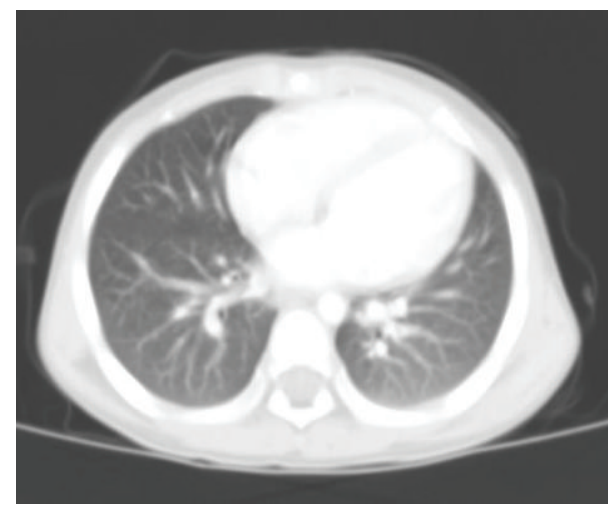

(b)

Figure 3: Thoracic CT reveals reticulonodular shadows in the lung window (a); after five weeks of treatment, thoracic CT showed that the lesions of the lung were reduced (b).

\section{CD3/CD8/CD45/CD4}

\begin{tabular}{|lcc|}
\hline Lymph events & 2131 & \\
$\mathrm{CD}^{+} \%$ lymph & 54 & $\mathrm{Lo}$ \\
$\mathrm{CD}^{+} \mathrm{CD}^{+}$\%lymph & 9 & $\mathrm{Lo}$ \\
$\mathrm{CD}^{+} \mathrm{CD}^{+} \%$ lymph & 42 & \\
$\mathrm{CD}^{+} \mathrm{CD} 4^{+} \mathrm{CD} 8^{+} \%$ lymph & 0 & \\
$\mathrm{~T} \mathrm{H} / \mathrm{S}$ ratio & $4-43$ & $\mathrm{Hi}$ \\
\hline
\end{tabular}
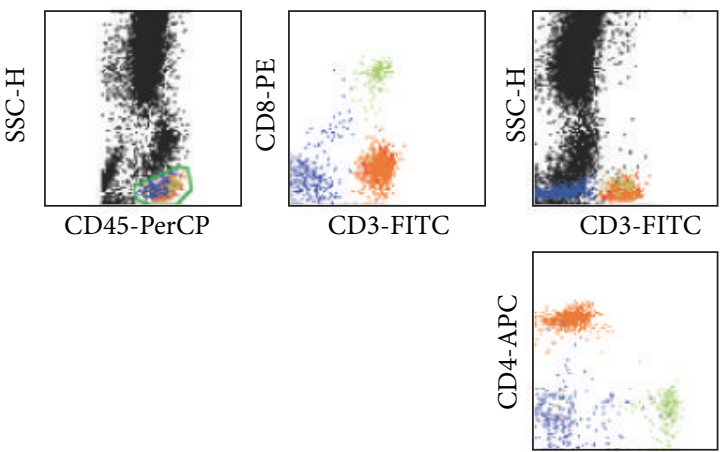

CD8-PE

$\mathrm{CD} 3 / \mathrm{CD}_{16}{ }^{+}$56/CD45/CD19

\begin{tabular}{|lcc|}
\hline Lymph events & 2175 & \\
$\mathrm{CD}^{+}$\%lymph & 54 & Lo \\
$\mathrm{CD} 16^{+} 56^{+} \%$ lymph & 2 & Lo \\
$\mathrm{CD}^{+} 9^{+}$\%lymph & 42 & Hi \\
\hline
\end{tabular}

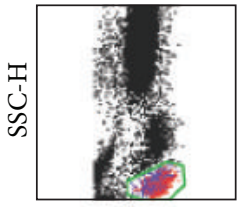

CD45-PerCP

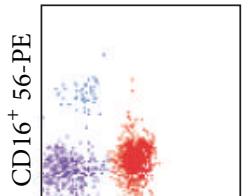

CD3-FITC

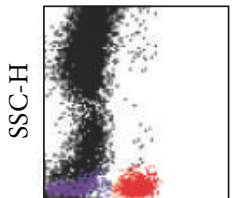

CD3-FITC

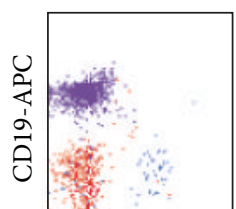

$\mathrm{CD}_{16}{ }^{+}$56-PE

FIGURE 4: Lymphocyte subset detection by flow cytometry, which showed that the percentages of CD3, CD3/CD $8^{+}, \mathrm{CD} 3 / \mathrm{CD} 4^{+}, \mathrm{CD} 19^{+}$, and NK were $54 \%, 9 \%, 42 \%, 42 \%$, and $2 \%$, respectively.

with a visible artificial round halo, and some yeast forms have characteristic narrow-based buds; sometimes, special PAS stains can also be useful for detecting the organisms. Therefore, using the characteristic features revealed by pathology, cryptococcal lymphadenitis is diagnosed easily. Once cryptococcosis is positively diagnosed, immediate antifungal treatment is required. Intravenous amphotericin B and oral fluconazole are the most common therapies, but sometimes oral flucytosine is added [18].

Our case suggests that the diagnosis of cryptococcal lymphadenitis is very difficult without etiology and pathology. If both abnormal lymph nodes in the neck and inflammation in the lungs are confirmed in a child, cryptococcal lymphadenitis should be considered, even with a negative culture result. 


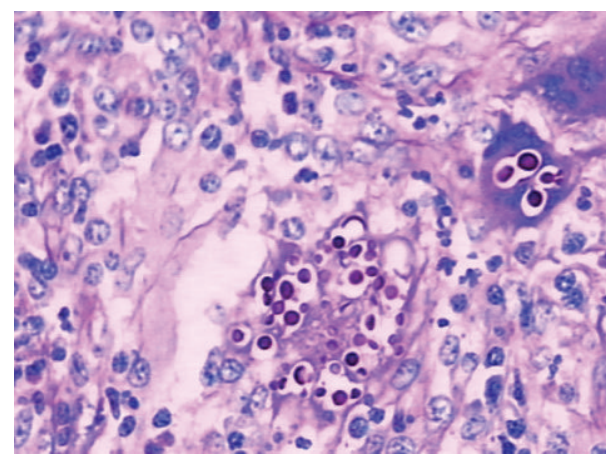

FIGURE 5: The cervical lymph node excisional biopsy revealed a lymphohistiocytic background with several blue, spherical structures that were surrounded by clear halos, which are indicative of Cryptococcus.

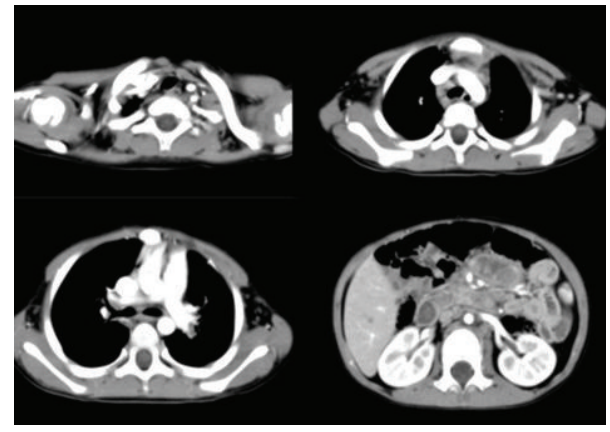

FIGURE 6: After five weeks of treatment, the thoracic and abdominal CT showed that the swelling of the lymph nodes was markedly diminished.

Lymph node biopsy should be performed, and if the diagnosis is established, antifungal treatment must be started as soon as possible to prevent complications related to cryptococcosis.

\section{Authors' Contribution}

Fengchang Bao and Hongna Tan contributed equally to this report.

\section{References}

[1] J. Diebold, C. Marche, and J. Audouin, "Lymph node modification in patients with the acquired immunodeficiency syndrome (AIDS) or with AIDS related complex (ARC). A histological, immuno-histopathological and ultrastructural study of 45 cases," Pathology Research and Practice, vol. 180, no. 6, pp. 590611, 1985.

[2] C. Ginsburg, L. Aaron, A. Lesueur, D. Salmon-Ceron, and D. Sicard, "Ganglion cryptoccososis in an AIDS patient on longterm fluconazole: relapse or cure?" Presse Medicale, vol. 26, no. 22, p. 1049, 1997.

[3] K. A. Sepkowitz, "Effect of HAART on natural history of AIDSrelated opportunistic disorders," The Lancet, vol. 351, no. 9098, pp. 228-230, 1998.

[4] M. Lanzafame, M. Trevenzoli, G. Carretta, L. Lazzarini, S. Vento, and E. Concia, "Mediastinal lymphadenitis due to cryptococcal infection in HIV-positive patients on highly active antiretroviral therapy," Chest, vol. 116, no. 3, pp. 848-849, 1999.

[5] D. Moukassa, X. Leroy, J. R. Ibara, B. Mounkassa, A. TsimbaNsonda, and J. Kokolo, "Cryptococcus neoformans granulomatous and necrosing lymphadenitis in an HIV-infected patient," Presse Medicale, vol. 28, no. 37, pp. 2039-2040, 1999.

[6] L. Putignani, G. Antonucci, M. G. Paglia et al., "Cryptococcal lymphadenitis as a manifestation of immune reconstitution inflammatory syndrome in an HIV-positive patient: a case report and review of the literature," International Journal of Immunopathology and Pharmacology, vol. 21, no. 3, pp. 751-756, 2008.

[7] E. Oscar Tapia, H. Miguel Villaseca, and O. Juan Carlos Araya, "Mesenteric cryptococcal lymphadenitis. Report of one case," Revista Medica de Chile, vol. 138, no. 12, pp. 1535-1538, 2010.

[8] E. Natukunda, V. Musiime, F. Ssali, H. Kizito, C. Kityo, and P. Mugyenyi, "A case of cryptococcal lymphadenitis in an HIVinfected child," AIDS Research and Human Retroviruses, vol. 27, no. 4, pp. 373-376, 2011.

[9] S. H. Kim, S. D. Kim, H. R. Kim et al., "Intraabdominal cryptococcal lymphadenitis in a patient with systemic lupus erythematosus," Journal of Korean Medical Science, vol. 20, no. 6, pp. 1059-1061, 2005.

[10] T. G. Mitchell and J. R. Perfect, "Cryptococcosis in the era of AIDS-100 years after the discovery of Crypotcoccus neoformans," Clinical Microbiology Reviews, vol. 8, no. 4, pp. 515-548, 1995.

[11] M. Mitha, P. Naicker, and P. Mahida, "Disseminated cryptococcosis in an HIV-negative patient in South Africa: the elusive differential diagnosis," Journal of Infection in Developing Countries, vol. 4, no. 8, pp. 526-529, 2010.

[12] B. A. Bilodeau and K. L. Fessele, "Non-Hodgkin's lymphoma," Seminars in Oncology Nursing, vol. 14, no. 4, pp. 273-283, 1998.

[13] S. Cole and K. Dunne, "Hodgkin's lymphoma," Nursing standard, vol. 18, no. 19, pp. 46-54, 2004.

[14] J. M. Fontanilla, A. Barnes, and C. F. von Reyn, "Current diagnosis and management of peripheral tuberculous Lymphadenitis," Clinical Infectious Diseases, vol. 53, no. 6, pp. 555-562, 2011.

[15] K. R. Steingart, L. L. Flores, N. Dendukuri et al., "Commercial Serological tests for the diagnosis of active pulmonary and extrapulmonary tuberculosis: an updated systematic review and Meta-Analysis," PLoS Medicine, vol. 8, no. 8, Article ID e1001062, 2011.

[16] K. S. Gustafson and L. Feldman, "Cryptococcal lymphadenitis diagnosed by fine-needle aspiration biopsy," Diagnostic Cytopathology, vol. 35, no. 2, pp. 103-104, 2007.

[17] R. Srinivasan, N. Gupta, R. Shifa, P. Malhotra, A. Rajwanshi, and A. Chakrabarti, "Cryptococcal lymphadenitis diagnosed by fine needle aspiration cytology: a review of 15 cases," Acta Cytologica, vol. 54, no. 1, pp. 1-4, 2010.

[18] M. Ritter and D. L. Goldman, "Pharmacotherapy of cryptococcosis," Expert Opinion on Pharmacotherapy, vol. 10, no. 15, pp. 2433-2443, 2009. 


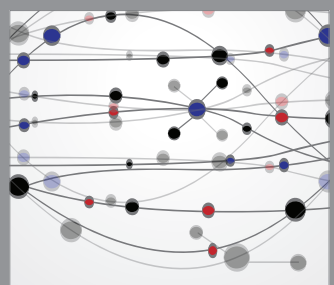

The Scientific World Journal
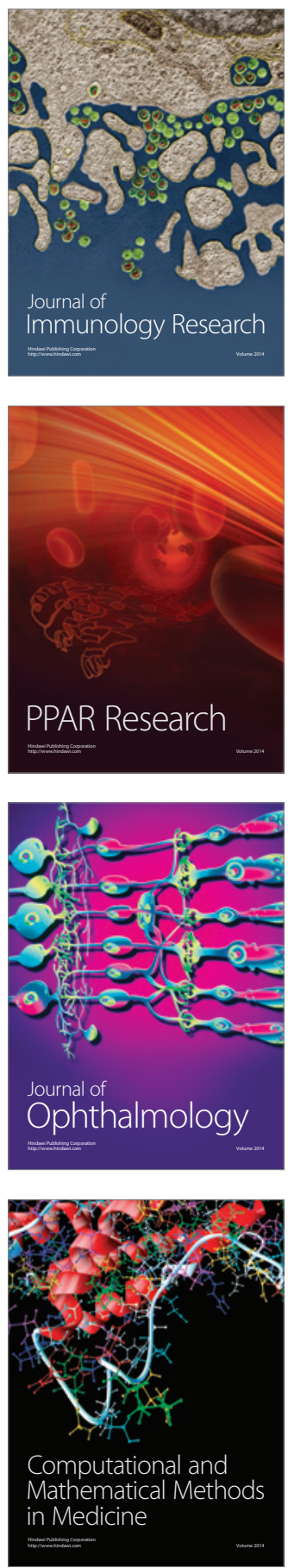

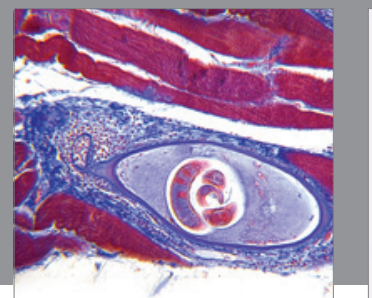

Gastroenterology

Research and Practice
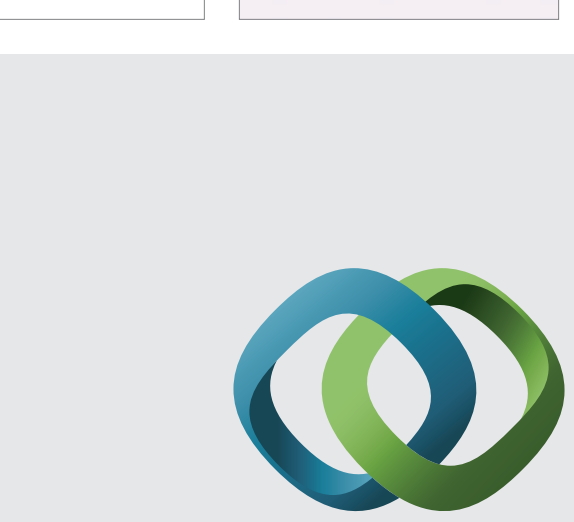

\section{Hindawi}

Submit your manuscripts at

http://www.hindawi.com
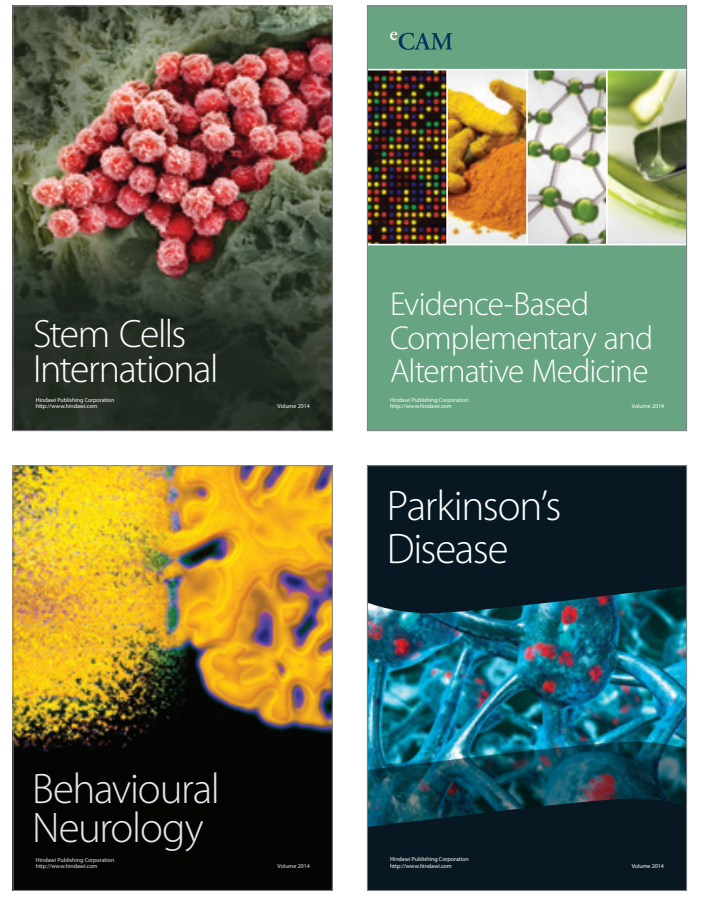
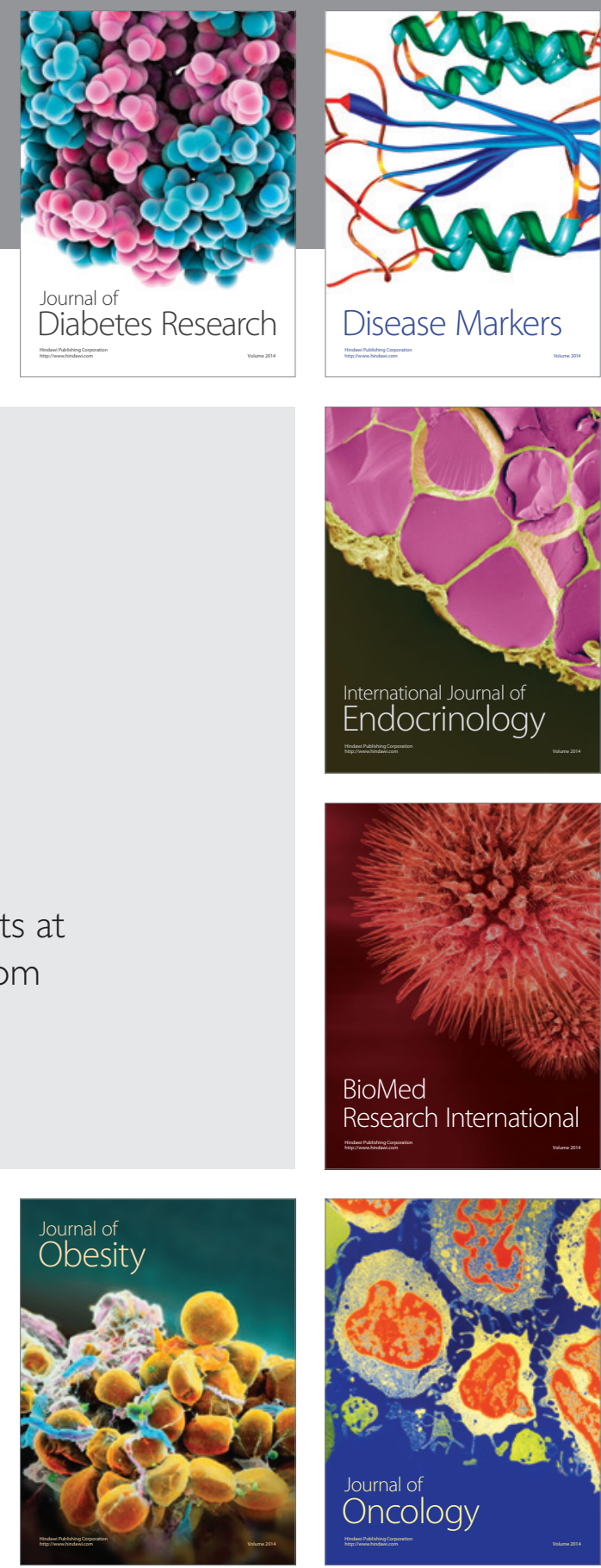

Disease Markers
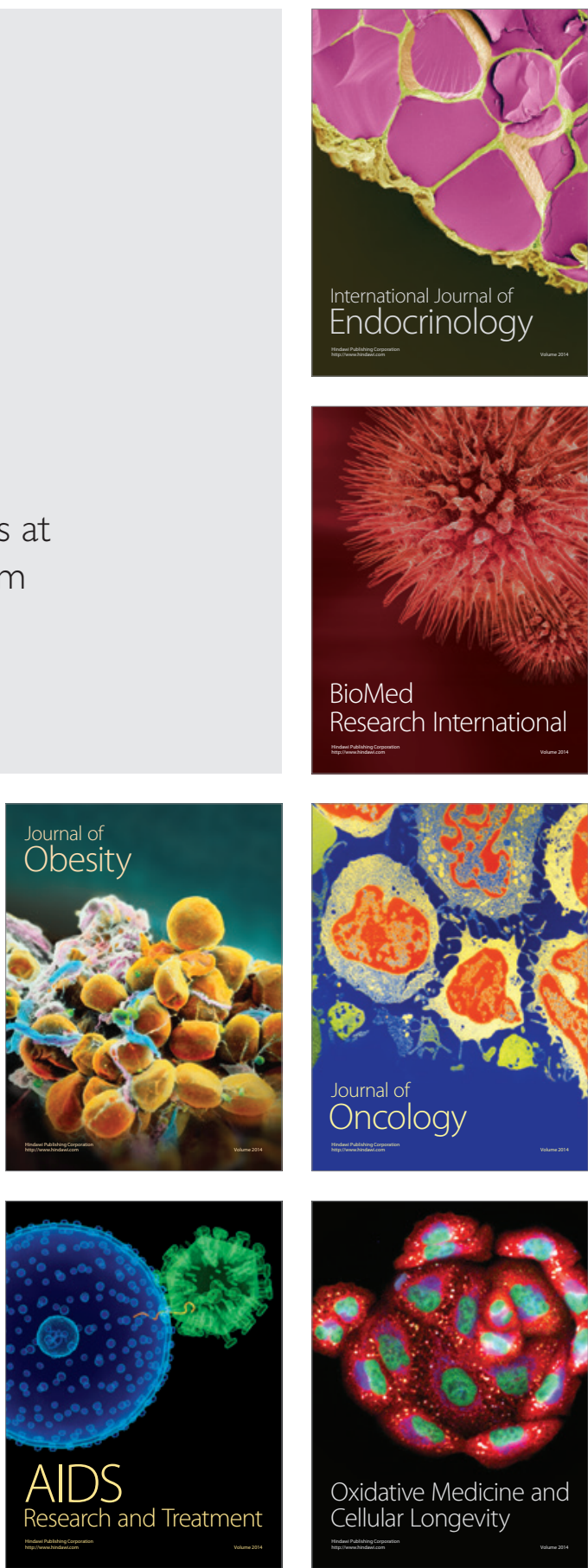